\title{
Distribution and Productivity of Naturalized Alfalfa in Mixed-Grass Prairie
}

\author{
Lan Xu1 ${ }^{*}$, Arvid Boe ${ }^{2}$, Patricia S. Johnson ${ }^{3}$, Roger N. Gates ${ }^{4}$, Christopher G. Misar ${ }^{5}$ \\ ${ }^{1}$ Department of Natural Resource Management, South Dakota State University, Brookings, SD, USA \\ ${ }^{2}$ Department of Agronomy, Horticulture, and Plant Science, South Dakota State University, Brookings, SD, USA \\ ${ }^{3}$ Department of Natural Resource Management, South Dakota State University, West River Ag Center, Rapid City, SD, USA \\ ${ }^{4}$ College of Agricultural and Environmental Sciences, Whitfield County Extension, University of Georgia, Dalton, GA, USA \\ ${ }^{5}$ USDA-ARS Northern Crop Science Laboratory, Fargo, ND, USA \\ Email: ^lan.xu@sdstate.edu, arvid.boe@sdstate.edu, patricia.johnson@sdstate.edu, Roger.gates@uga.edu, \\ Christopher.Misar@ARS.USDA.GOV
}

How to cite this paper: $\mathrm{Xu}, \mathrm{L}$., Boe, A., Johnson, P.S., Gates, R.N. and Misar, C.G. (2019) Distribution and Productivity of Naturalized Alfalfa in Mixed-Grass Prairie. American Journal of Plant Sciences, 10, 1030-1047.

https://doi.org/10.4236/ajps.2019.106075

Received: May 5, 2019

Accepted: June 22, 2019

Published: June 25, 2019

Copyright $\odot 2019$ by author(s) and Scientific Research Publishing Inc. This work is licensed under the Creative Commons Attribution International License (CC BY 4.0).

http://creativecommons.org/licenses/by/4.0/

\begin{abstract}
Alfalfa (Medicago sativa L.) is an important forage and conservation crop in North America but occurrences of naturalized alfalfa in rangelands are rare. A naturalized population of yellow-flowered alfalfa in mixed-grass prairie on the Grand River National Grassland in South Dakota has potential agricultural value for the region. Despite this value, the distribution pattern of this alfalfa among and within native plant communities in the northern Great Plains is unknown. Field studies were conducted from 2003 through 2006 along topographic positions at two sites where yellow-flowered alfalfa was naturalized to evaluate the relationships of yellow-flowered alfalfa on biomass production, cover, species diversity, and alfalfa seedbank distribution characteristics. High yellow-flowered alfalfa cover $(>50 \%)$ was associated with increased total biomass and occurred exclusively in swales and toeslopes that had silty loam soils. However, species diversity and non-alfalfa biomass were reduced when yellow-flowered alfalfa cover was high. Yellow-flowered alfalfa cover was lower and species richness was higher on backslopes and shoulder areas where sandy loam soils were present. A strong positive linear association existed between yellow-flowered alfalfa cover and alfalfa seedbank density ( $\mathrm{r}$ values ranged from 0.76 to $0.82, P<0.0001$ ). Greater than $99 \%$ of the seeds were viable but germination rate was only $4 \%$, indicating a high percentage of hard seed in this alfalfa population. Naturalized yellow-flowered alfalfa was a dominant component of a stable, low diversity plant community composed of mainly introduced species in rich soils of swales/toeslopes that contributed to forage production and quality in mixed-grass prairie in the northern Great Plains.
\end{abstract}




\section{Keywords}

Biomass Production, Diversity, Falcata, Lucerne, Rangelands, Seed Bank, Species Richness

\section{Introduction}

Alfalfa (Medicago sativa L.) is a major component of feed for livestock and is one of the most productive forage species in North America. Interseeding alfalfa in grassland can be an effective approach for increasing forage and livestock production in semiarid regions [1] [2] [3] [4]. Alfalfa is indigenous to regions of Asia characterized by a continental climate [5]. Alfalfa has been planted on millions of hectares over the past 100 years in North America since its successful introduction in the 1860s. However, incidents of alfalfa becoming naturalized in North American rangelands are rare [6].

Yellow-flowered alfalfa (Medicago sativa L. ssp. falcata [L.] Arcang.) (hereafter YFA) occurs naturally at latitudes up to $64^{\circ} \mathrm{N}$ in Siberia, and is adapted to the climatic conditions of the northern Great Plains of the USA [7]. It is more winter-hardy, drought tolerant, and grazing tolerant than other alfalfas because of proliferation of adventitious shoots from roots, deep-set crown and fibrous root system [7]-[12]. Slow regrowth following defoliation improves grazing tolerance [9] [13].

Smith [2] described substantial benefits of YFA (which he referred to as "falcata") to livestock production on his ranch in northwestern South Dakota. Mechanical interseeding and subsequent natural reseeding of "falcata" on his ranch over several decades resulted in dramatic increases in forage production and quality [2], with coincident improved soil carbon and nitrogen without elevation of nitrous oxide emissions [3] [14] [15].

Richardson, et al. [16] reported that naturalization often refers to "alien plants that reproduce consistently and sustain populations over many life cycles without direct intervention by humans (or in spite of human intervention); they often recruit offspring freely, usually close to adult plants, and do not necessarily invade natural, seminatural or human-made ecosystems." However, spread of naturalized YFA from private land (i.e., the Smith Ranch) to adjacent USDA Forest Service managed land in mixed-grass prairie on the Grand River National Grassland (GRNG) presents a potential dilemma. Alfalfa is generally not considered to be invasive and incidence of natural propagation on rangelands is infrequent. Occurrence of a population able to reproduce naturally in native rangeland demonstrates genetic potential for rehabilitating severely depleted rangeland ecosystems. Conversely, characteristics that make it suitable for rangeland improvement might be expected to increase its invasive potential [17]. For example, crested wheatgrass (Agropyron cristatum [L.] Gaertn.) has historically been controversial because of similar characteristics [18]. 
While there is evidence to demonstrate the forage value of YFA [2] [3] [4] [12] [19], its impact on native plant communities and potential threat to native biological diversity are unknown. In addition, it is unclear how naturalized YFA populations spread in native plant communities. These critical deficiencies reduce our ability to properly utilize and manage YFA in semiarid rangelands. The goal of this study was to evaluate biomass production and distribution patterns of naturalized YFA. Our objectives were to determine: 1) spatial distribution patterns of naturalized YFA along environmental gradients, 2) association between naturalized YFA and species diversity and biomass production along those gradients, and 3) spatial distribution, density, and viability of the soil seed bank of naturalized YFA.

\section{Materials and Methods}

\subsection{Study Site}

This study was conducted in Pasture 4B on the GRNG (US Forest Service Dakota Prairie Grasslands) in northwestern South Dakota, USA (lat. $45^{\circ} 49^{\prime} 21^{\prime \prime N}$, long. $\left.102^{\circ} 33^{\prime} 04^{\prime \prime} \mathrm{W}\right)$. The GRNG comprises 62,753 hectares of public land with parcels of private land interspersed throughout. The topography is characterized by rolling hills, river breaks, and scattered buttes and badlands [20]. Climate is semiarid with high temporal variation in precipitation and high frequency of drought. Mean annual precipitation is $405 \mathrm{~mm}$ and mean annual temperature is $6.11^{\circ} \mathrm{C}$. Extreme daily high and low temperature records (1908-2012) are $45^{\circ} \mathrm{C}$ and $-43^{\circ} \mathrm{C}$, respectively [21] [22]. Soil types range from sandy loam on backslopes and uplands to clay loam in swales and drainage areas [23]. Natural vegetation is mixed-grass prairie with a variety of species including western wheatgrass (Pascopyrum smithii [Rydb.] Á. Löve), green needlegrass (Nassella viridula [Trin.] Barkworth), threadleaf sedge (Carex filifolia Nutt.), blue grama (Bouteloua gracilis [Willd. ex Kunth] Lag. ex Griffiths), buffalograss (Bouteloua dactyloides [Nutt.] J. T. Columbus), purple coneflower (Echinacea angustifolia DC.), silverleaf Indian breadroot (Pediomelum argophylla [Pursh] J. Grimes) and purple prairie clover (Dalea purpurea Vent.) [24]. Most of the GRNG is permitted for grazing cattle (Bos taurus L.) through the Grand River Cooperative Grazing Association [25].

In June 2003, reconnaissance of Pasture 4B identified two sites where YFA appeared to be naturalized based on variation in plant size and age. These sites, designated north $(\mathrm{N})$ and south $(\mathrm{S})$, were selected because they were representative of the distribution of YFA across environmental gradients in Pasture 4B on the GRNG. Two permanent transects were established for the purpose of vegetation sampling on each site. On the $\mathrm{N}$ site, each transect traversed a gradient from the top of a side-shoulder [23] through a swale to the top of the opposite side-shoulder. On the S site, each transect began on the top of a side-shoulder and terminated on a toeslope at the shoreline of a small perennial stream. Transect lengths ranged from $108-130 \mathrm{~m}$; change in elevation from highest to lowest 
points was $2 \mathrm{~m}$ on the $\mathrm{N}$ transects and $7 \mathrm{~m}$ on the $\mathrm{S}$ transects. Three distinct plant communities were recognized along each transect. For the $\mathrm{N}$ site, the plant communities were threadleaf sedge (Sedge), alfalfa-crested wheatgrass (Alfalfa-Crested), and alfalfa (Alfalfa). For the S site, the plant communities were threadleaf sedge-green needlegrass (Sedge-needlegrass), crested wheatgrass-alfalfa (Crested-Alfalfa), and alfalfa (Alfalfa).

\subsection{Vegetation Sampling}

Ocular estimates of cover were made for each species in $2 \mathrm{~m} \times 1 \mathrm{~m}$ quadrats at 2 $\mathrm{m}$ intervals along each transect. The same cover plots were sampled in 2003 and 2004. Biomass was obtained by clipping three $1.45 \mathrm{~m} \times 0.3 \mathrm{~m}$ quadrats randomly located along each transect within each plant community. Areas used to estimate cover were avoided. Aboveground vegetation within each biomass quadrat was clipped to the ground and sorted into two categories: alfalfa and other species. Biomass was oven-dried at $60^{\circ} \mathrm{C}$ for 72 hours and weighed. New biomass plots were selected in each year. Sampling dates in 2003 were $26-27$ June (N site) and 17 July (S site). In 2004, sampling dates were $25-27$ June for both $\mathrm{N}$ and S sites. Plant species were identified in the field during vegetation sampling. Specimens of unknown species were collected, identified and verified later using The C. A. Taylor Herbarium at South Dakota State University. Nomenclature for all plant species in this study follows the USDA Plants Database [26].

\subsection{Soil Sampling}

Three soil cores ( $2 \mathrm{~cm}$ dia.) were collected from each biomass plot and separated into $0-15 \mathrm{~cm}$ and $15-30 \mathrm{~cm}$ depths in both 2003 and 2004 (same dates as for biomass above). Soil texture was determined by South Dakota State University Soil Testing Laboratory, using standard laboratory procedures [27].

\subsection{Seed Bank Density, Germination, and Viability}

Three soil samples were extracted from each biomass quadrat using a bulb planter $(5 \mathrm{~cm}$ dia. $\times 7.5 \mathrm{~cm}$ depth) in June 2004, November 2005, and June 2006. Alfalfa seeds were extracted from soil using a series of sieves, counted, and verified. Seed germination and viability were determined by the SDSU Seed Technology Testing Laboratory using AOSA protocol for alfalfa [28]. Four 100-seed replicates were tested per site per year.

\subsection{Statistical Analyses}

Based on YFA cover estimates and modified Daubenmire's cover class method [29], individual quadrats $(n=148)$ for both $N$ and S sites were arbitrarily categorized into one of three classes (Class I $=0 \%$ alfalfa, $n=72$; Class II $=0.1$ to $50 \%$ alfalfa, $\mathrm{n}=42$; and Class III $>50 \%$ alfalfa, $\mathrm{n}=34$ ). Species richness [30] for total species, graminoids, forbs, native species, introduced species, perennial species, and annual species were determined for each cover quadrat.

Shannon-Wiener indices for species diversity were calculated for comparison 
among the three alfalfa cover classes [31]:

$$
H^{\prime}=-\sum_{i=1}^{S} p_{i} \ln p_{i}
$$

where $P_{i}$ represented the proportion of total cover contributed by each species. Species evenness was also calculated:

$$
J^{\prime}=H^{\prime} / \ln S
$$

where $S$ represents species richness. The proportional cover of each species was determined for each alfalfa cover class and used to create rank abundance curves [31] for illustrating species diversity impact by alfalfa cover.

All statistical analyses were conducted using procedures in SAS [32]. A subset of species was selected using a stepwise procedure (PROC STEPDISC) as input for Canonical Discriminant Analysis (CDA) (PROC CANDISC). CDA was used to evaluate the association of the spatial distribution of YFA and other species with topographic position (i.e., shoulder, backslope, swale/toeslope) [23].

One-way Analysis of Variance using PROC ANOVA determined the contribution of cover class to variation of species richness and biomass. Levene's test was used for testing homogeneity of variance. Linear regression was performed to determine the relationship between YFA cover and alfalfa seedbank density.

\section{Results}

Distinct plant assemblages were associated with topographic positions (Figure 1). The first canonical variate accounted for $79.7 \%$ of the variation of species cover and separated the shoulder from swale ( $\mathrm{N}$ site) and toeslope ( $\mathrm{S}$ site) communities. The primary determinants of canonical variate 1 were threadleaf sedge and YFA cover. Vegetation on the shoulders was dominated by threadleaf sedge and sub-dominated by prairie sandreed (Calamovilfa longifolia [Hook]. Scribn.). Threadleaf sedge occurred only on the shoulder, where soils were predominantly sandy loam. YFA occurred only rarely on the shoulder and upper backslope. Vegetation on the toeslope area (S site), where soils were mostly silty loam, was dominated by YFA and western wheatgrass. Canonical variate 2 accounted for an additional $20.3 \%$ of the variation and distinguished the backslope community. The plant community on the backslope shared species with shoulder and swale/toeslope communities but was distinguished by needlegrass cover.

A total of 76 species were found in the study, including 18 graminoids, 55 forbs, 1 shrub and 3 succulents (Table 1(a) and Table 1(b)). We found a total of 69 species in 2003 and 63 species in 2004. Of the 76 species, 14 species were found only in 2003 and 7 only in 2004. Species richness was not different between YFA cover Classes I and II (Figure 2). However, species richness was approximately $50 \%$ less when YFA cover was greater than 50\% (i.e., Class III) (Figure 2). Graminoid, forb, perennial, and annual species richness values exhibited similar patterns. Notably, native species richness declined as YFA cover increased. Species diversity and evenness significantly decreased as YFA cover increased (Figure 3). 


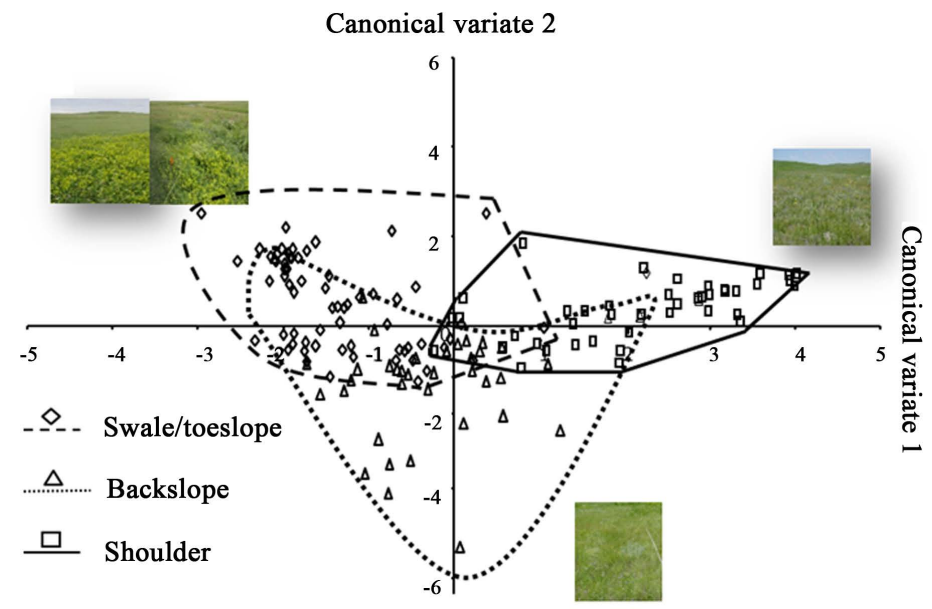

(a)

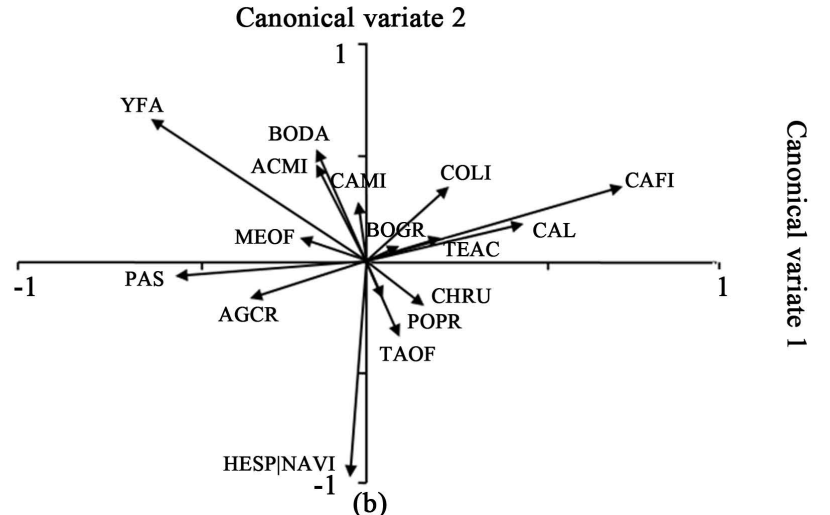

Figure 1. Canonical Discriminant Analysis ordination showed spatial vegetation distribution. Communities were sampled along topographic position gradient from Shoulder, Backslope, and Swale/toeslope (a) and species composition (b) was associated with topography along ordination Canonical Variate 1 and 2. In panel (b), each four-letter code refers to the first two letters of the genus and species for a plant species, except for YFA = Predominantly yellow-flowered alfalfa and HESP|NAVI = Hesperostipa comata and Hesperostipa spartea|Nassella viridula; where BODA = Bouteloua dactyloides, BOGR = Bouteloua gracilis, ACMI = Achillea millefolium, CAMI = Camelina microcarpa, $\mathrm{COLI}=$ Collomia linearis, TEAC $=$ Tetraneuris acaulis, $\mathrm{CALO}=$ Calamovilfa longifolia, CAFI $=$ Carex filifolia, $\mathrm{CHRU}$ = Chenopodium rubrum, POPR $=$ Poa pratensis, TAOF $=$ Taraxacum officinalis, AGCR = Agropyron cristatum, PASM = Pascopyrum smithii, $\mathrm{MEOF}=$ Melilotus officinalis .

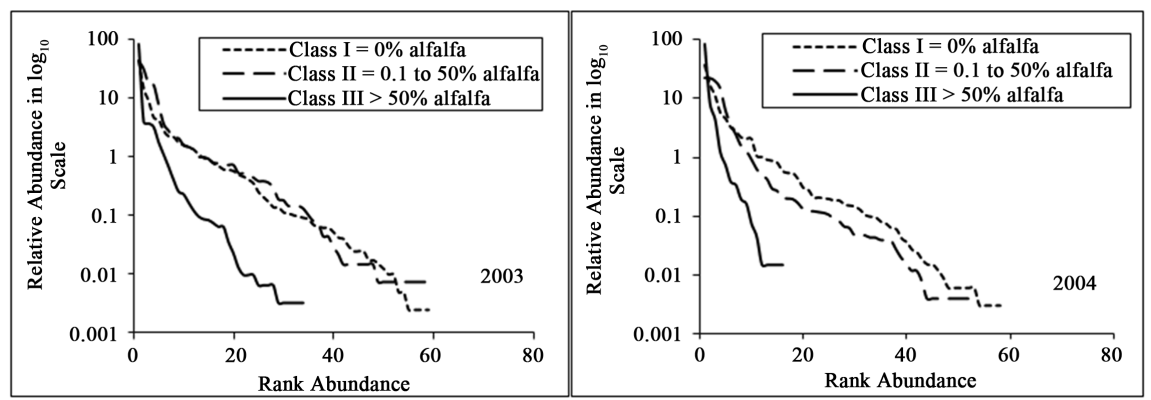

Figure 2. Change in relative abundance of plant species under different alfalfa cover classes in the Grand River National Grassland near Lodgepole, SD, illustrating plant species diversity was significantly reduced in high alfalfa cover $(>50 \%)$ with short length (lower species richness) and steep slope (less evenness). 
Table 1. (a) Cover and frequency of plant species for three alfalfa cover classes on Pasture 4B of the Grand River National Grassland near Lodgepole, SD in 2003. Non-native species denoted by an asterisk ${ }^{*}$ ); (b) Cover and frequency of plant species for three alfalfa cover classes on Pasture 4B of the Grand River National Grassland near Lodgepole, SD in 2004. Non-native species denoted by an asterisk $\left(^{*}\right)$.

\begin{tabular}{|c|c|c|c|c|c|c|c|c|}
\hline \multirow[b]{2}{*}{ Scientific Name } & \multirow[b]{2}{*}{$\begin{array}{l}\text { Life } \\
\text { Form }^{\mathrm{b}}\end{array}$} & \multirow[b]{2}{*}{$\begin{array}{l}\text { Life } \\
\text { Span }^{c}\end{array}$} & \multicolumn{2}{|c|}{ Class I $^{\mathrm{a}}(\mathrm{n}=72)$} & \multicolumn{2}{|c|}{ Class II $(\mathrm{n}=42)$} & \multicolumn{2}{|c|}{ Class III $(\mathrm{n}=34)$} \\
\hline & & & $\begin{array}{l}\text { Frequency }^{\mathrm{d}} \\
\quad(\%)\end{array}$ & $\begin{array}{c}\text { Covere }^{e} \\
(\%)\end{array}$ & $\begin{array}{l}\text { Frequency } \\
(\%)\end{array}$ & $\begin{array}{l}\text { Cover } \\
(\%)\end{array}$ & $\begin{array}{l}\text { Frequency } \\
\quad(\%)\end{array}$ & $\begin{array}{c}\text { Cover } \\
(\%)\end{array}$ \\
\hline Medicago sativa ssp. falcata (L). Arcang. * & $\mathrm{F}$ & $\mathrm{P}$ & 0.00 & 0.00 & 100 & 14.39 & 100 & 78.15 \\
\hline Carex filifolia Nutt. & G & $\mathrm{P}$ & 81 & 24.59 & 55 & 5.55 & 12 & 0.15 \\
\hline Hesperostipa spp. ${ }^{f}$ & G & $\mathrm{P}$ & 78 & 2.99 & 60 & 0.99 & 24 & 0.08 \\
\hline Pascopyrum smithii (Rydb.) Á. Löve & G & $\mathrm{P}$ & 71 & 8.30 & 81 & 5.74 & 56 & 2.99 \\
\hline Taraxacum officinale F.H. Wigg * & $\mathrm{F}$ & $\mathrm{P}$ & 68 & 0.62 & 38 & 0.17 & 24 & 0.08 \\
\hline Chenopodium rubrum $\mathrm{L}$. & $\mathrm{F}$ & A & 61 & 0.34 & 76 & 0.26 & 44 & 0.21 \\
\hline Melilotus officinalis (L.) Lam. ${ }^{*}$ & $\mathrm{~F}$ & $\mathrm{~B} / \mathrm{P}$ & 51 & 1.57 & 67 & 2.55 & 44 & 1.64 \\
\hline Collomia linearis Nutt. & $\mathrm{F}$ & A & 50 & 2.42 & 36 & 0.69 & 12 & 0.07 \\
\hline Camelina microcarpa Andrz. ex. DC.* & $\mathrm{F}$ & A & 44 & 0.10 & 76 & 0.25 & 47 & 0.11 \\
\hline $\begin{array}{l}\text { Lactuca tatarica (L.) C.A. Mey. Var. pulchella } \\
\text { (Pursh) Breitung }\end{array}$ & $\mathrm{F}$ & A & 43 & 0.23 & 21 & 0.33 & 6 & 0.01 \\
\hline Plantago elongate Pursh & $\mathrm{F}$ & A & 39 & 0.05 & 17 & 0.06 & 12 & 0.01 \\
\hline Vicia Americana Muhl. ex Willd. & F & $\mathrm{P}$ & 38 & 0.26 & 47 & 0.16 & 35 & 0.37 \\
\hline $\begin{array}{l}\text { Bouteloua gracilis (Willd. ex Kunth) Lag. ex Grif- } \\
\text { fiths }\end{array}$ & G & $\mathrm{P}$ & 33 & 0.99 & 29 & 0.52 & 9 & 0.02 \\
\hline Agropyron cristatum (L.) Gaertn. ${ }^{*}$ & G & $\mathrm{P}$ & 29 & 5.06 & 62 & 10.41 & 53 & 3.43 \\
\hline Lappula occidentalis (S. Watson) Greene) & $\mathrm{F}$ & A & 29 & 0.03 & 14 & 0.01 & 9 & 0.01 \\
\hline Poa pratensis L. ${ }^{*}$ & G & $\mathrm{P}$ & 25 & 1.23 & 24 & 1.20 & 62 & 3.63 \\
\hline Pediomelum argophyllum (Pursh) J. Grimes & $\mathrm{F}$ & $\mathrm{P}$ & 25 & 0.04 & 14 & 0.06 & 3 & 0.00 \\
\hline Sphaeralcea coccinea (Nutt.) Rydb. & F & $\mathrm{P}$ & 25 & 0.51 & 26 & 0.33 & 9 & 0.01 \\
\hline $\begin{array}{c}\text { Oenothera suffrutescens }(\text { Ser.) W.L. Wagner \& } \\
\text { Hoch }\end{array}$ & $\mathrm{F}$ & $\mathrm{P}$ & 22 & 0.03 & 14 & 0.01 & - & - \\
\hline Achillea millefolium $\mathrm{L}$. & $\mathrm{F}$ & $\mathrm{P}$ & 21 & 0.90 & 52 & 0.91 & 59 & 1.01 \\
\hline Lygodesmia juncea (Pursh) D. Don ex Hook. & $\mathrm{F}$ & $\mathrm{P}$ & 21 & 0.06 & 5 & 0.00 & - & - \\
\hline Cirsium undulatum (Nutt.) Spreng. & $\mathrm{F}$ & $\mathrm{P}$ & 19 & 0.14 & 5 & $0.00^{\mathrm{g}}$ & 3 & 0.06 \\
\hline Calamovilfa longifolia (Hook.) Scribn. & G & $\mathrm{P}$ & 18 & 0.40 & 5 & 0.17 & - & - \\
\hline Oxytropis lambertii Pursh & $\mathrm{F}$ & $\mathrm{P}$ & 15 & 0.33 & 5 & 0.03 & - & - \\
\hline Phlox hoodia Richardson & $\mathrm{F}$ & $\mathrm{P}$ & 14 & 0.05 & 10 & 0.13 & 3 & 0.00 \\
\hline Artemisia frigida Willd. & $\mathrm{F}$ & $\mathrm{P}$ & 13 & 0.32 & 19 & 0.24 & 3 & 0.00 \\
\hline Artemisia ludoviciana Nutt. & $\mathrm{F}$ & $\mathrm{P}$ & 13 & 1.27 & 2 & 0.24 & 6 & 0.03 \\
\hline Hedeoma hispida Pursh & $\mathrm{F}$ & $\mathrm{P}$ & 13 & 0.01 & 10 & 0.01 & - & - \\
\hline Penstemon spp. & $\mathrm{F}$ & $\mathrm{P}$ & 13 & 0.02 & 12 & 0.03 & - & - \\
\hline Bromus arvensis L. ${ }^{*}$ & G & A & 11 & 0.06 & 26 & 0.05 & 6 & 0.01 \\
\hline Ellisia nyctelea (L.) L. & $\mathrm{F}$ & A & 11 & 0.08 & 2 & 0.00 & - & - \\
\hline
\end{tabular}




\section{Continued}

\begin{tabular}{|c|c|c|c|c|c|c|c|c|}
\hline $\begin{array}{c}\text { Vulpia octoflora (Walter) } \\
\text { Rydb. var. glauca (Nutt.) Fernald }\end{array}$ & G & A & 11 & 0.02 & - & - & - & - \\
\hline Tetraneuris acaulis (Pursh) Greene var. acaulis & $\mathrm{F}$ & A & 11 & 0.12 & - & - & - & - \\
\hline Opuntia fragilis (Nutt.) Haw & $\mathrm{F}$ & $\mathrm{P}$ & 11 & 0.04 & 5 & 0.00 & - & - \\
\hline Allium textile A. Nelson \& J. F. Macbr. & $\mathrm{F}$ & $\mathrm{P}$ & 10 & 0.01 & 7 & 0.01 & - & - \\
\hline Descurainia pinnata (Walter) Britton & $\mathrm{F}$ & A & 10 & 0.08 & 10 & 0.13 & 3 & 0.06 \\
\hline Eriogonum flavum Nutt. & $\mathrm{F}$ & A & 10 & 0.01 & 7 & 0.01 & - & - \\
\hline Liatris punctata Hook. & $\mathrm{F}$ & $\mathrm{P}$ & 10 & 0.05 & 2 & 0.00 & - & - \\
\hline Artemisia dracunculus $\mathrm{L}$. & $\mathrm{F}$ & $\mathrm{P}$ & 8 & 0.20 & 5 & 0.15 & - & - \\
\hline Bouteloua dactyloides (Nutt.) J.T. Columbus & G & $\mathrm{P}$ & 8 & 0.54 & 12 & 0.50 & 3 & 0.24 \\
\hline Echinacea angustifolia DC. & $\mathrm{F}$ & $\mathrm{P}$ & 8 & 0.03 & 5 & 0.12 & - & - \\
\hline $\begin{array}{l}\text { Lotus unifoliolatus (Hook.) } \\
\text { Benth. var. unifoliolatus }\end{array}$ & $\mathrm{F}$ & A & 8 & 0.76 & - & - & - & - \\
\hline Plantago patagonica Jacq. & $\mathrm{F}$ & A & 8 & 0.01 & 14 & 0.05 & 9 & 0.01 \\
\hline Thlaspi arvense $\mathrm{L} . *$ & $\mathrm{~F}$ & A & 7 & 0.01 & 2 & 0.00 & - & - \\
\hline Dalea purpurea Vent. & $\mathrm{F}$ & $\mathrm{P}$ & 6 & 0.02 & - & - & - & - \\
\hline Erysimum asperum (Nutt.) DC. & $\mathrm{F}$ & $\mathrm{B}$ & 6 & 0.02 & 2 & 0.00 & - & - \\
\hline Koeleria macrantha (Ledeb.) Schult. & G & $\mathrm{P}$ & 6 & 0.01 & 2 & 0.00 & 3 & 0.00 \\
\hline Tragopogon dubius Scop. ${ }^{*}$ & $\mathrm{~F}$ & $\mathrm{~B}$ & 6 & 0.06 & 21 & 0.04 & - & - \\
\hline Antennaria sp. & $\mathrm{F}$ & $\mathrm{P}$ & 3 & 0.00 & - & - & - & - \\
\hline Astragalus crassicarpus Nutt. & $\mathrm{F}$ & $\mathrm{P}$ & 3 & 0.00 & - & - & - & - \\
\hline Rosa woodsii Lindl. & $\mathrm{S}$ & $\mathrm{P}$ & 3 & 0.28 & 10 & 0.10 & - & - \\
\hline Viola nuttallii Pursh & $\mathrm{F}$ & $\mathrm{P}$ & 3 & 0.01 & 2 & 0.00 & - & - \\
\hline Heterotheca pumila (Greene) Semple & $\mathrm{F}$ & $\mathrm{P}$ & 1 & 0.01 & - & - & - & - \\
\hline Convolvulus arvensis $\mathrm{L}^{*}{ }^{*}$ & $\mathrm{~F}$ & $\mathrm{P}$ & 1 & 0.00 & 5 & 0.00 & 6 & 0.01 \\
\hline Escobaria missouriensis (Sweet) D.R. Hunt & $\mathrm{F}$ & $\mathrm{P}$ & 1 & 0.00 & - & - & - & - \\
\hline Distichlis spicata Greene & G & $\mathrm{P}$ & 1 & 0.00 & 12 & 0.43 & 9 & 0.62 \\
\hline Polygala alba Nutt. & $\mathrm{F}$ & $\mathrm{P}$ & 1 & 0.00 & - & - & - & - \\
\hline Potentilla pensylvanica L. & $\mathrm{F}$ & $\mathrm{P}$ & 1 & 0.00 & 2 & 0.02 & - & - \\
\hline Solidago sp. & $\mathrm{F}$ & $\mathrm{P}$ & 1 & 0.83 & 2 & 0.05 & 3 & 0.00 \\
\hline Grindelia squarrosa (Pursh) Dunal & $\mathrm{F}$ & $\mathrm{P}$ & - & - & 5 & 0.00 & - & - \\
\hline Pediomelum esculentum (Pursh) Rydb. & $\mathrm{F}$ & $\mathrm{P}$ & - & - & 5 & 0.00 & - & - \\
\hline Hordeum jubatum L. & G & $\mathrm{P}$ & - & - & 2 & 0.00 & 3 & 0.00 \\
\hline Agoseris glauca (Pursh) Raf. & $\mathrm{F}$ & $\mathrm{P}$ & - & - & 2 & 0.00 & - & - \\
\hline Bromus inermis Leyss. ${ }^{*}$ & G & $\mathrm{P}$ & - & - & 2 & 0.00 & - & - \\
\hline Lithospermum incisum Lehm & $\mathrm{F}$ & $\mathrm{P}$ & - & - & 2 & 0.00 & - & - \\
\hline Mertensia lanceolate (Pursh) DC. & $\mathrm{F}$ & $\mathrm{P}$ & - & - & 2 & 0.00 & - & - \\
\hline $\begin{array}{l}\text { Mirabilis laevis (Benth.) } \\
\text { Curran var. villosa (Kellogg) Spellenb. }\end{array}$ & $\mathrm{F}$ & $\mathrm{P}$ & - & - & 2 & 0.00 & - & - \\
\hline Salsola iberica L. ${ }^{*}$ & $\mathrm{~F}$ & A & - & - & 2 & 0.00 & - & - \\
\hline
\end{tabular}


(b)

\begin{tabular}{|c|c|c|c|c|c|c|c|c|}
\hline \multirow[b]{2}{*}{ Scientific Name } & \multirow{2}{*}{$\begin{array}{l}\text { Life } \\
\text { Form }^{\mathrm{b}}\end{array}$} & \multirow{2}{*}{$\begin{array}{l}\text { Life } \\
\text { Span }^{c}\end{array}$} & \multicolumn{2}{|c|}{ Class I $^{\mathrm{a}}(\mathrm{n}=72)$} & \multicolumn{2}{|c|}{ Class II $(n=42)$} & \multicolumn{2}{|c|}{ Class III $(\mathrm{n}=34)$} \\
\hline & & & $\begin{array}{l}\text { Frequency } \\
\quad(\%)\end{array}$ & $\begin{array}{c}\text { Cover }^{\mathrm{e}} \\
(\%)\end{array}$ & $\begin{array}{l}\text { Frequency } \\
\quad(\%)\end{array}$ & $\begin{array}{l}\text { Cover } \\
(\%)\end{array}$ & $\begin{array}{l}\text { Frequency } \\
\quad(\%)\end{array}$ & $\begin{array}{c}\text { Cover } \\
(\%)\end{array}$ \\
\hline Medicago sativa ssp. falcata (L). Arcang.* & $\mathrm{F}$ & $\mathrm{P}$ & 0.00 & 0.00 & 100 & 11.79 & 100 & 71.75 \\
\hline Pascopyrum smithii (Rydb.) Á. Löve & G & $P$ & 80 & 10.03 & 69 & 11.89 & 50 & 1.04 \\
\hline Melilotus officinalis (L.) Lam. ${ }^{*}$ & $\mathrm{~F}$ & B & 61 & 1.89 & 76 & 1.93 & 37 & 0.04 \\
\hline Carex filifolia Nutt. & G & $\mathrm{P}$ & 59 & 21.68 & 35 & 7.86 & - & - \\
\hline Koeleria macrantha (Ledeb.) Schult. & G & $\mathrm{P}$ & 37 & 0.58 & 22 & 0.34 & - & - \\
\hline Plantago elongate Pursh & $\mathrm{F}$ & A & 37 & 1.27 & 22 & 0.83 & 38 & 0.63 \\
\hline Agropyron cristatum (L.) Gaertn. ${ }^{*}$ & G & $\mathrm{P}$ & 36 & 6.53 & 63 & 0.53 & 88 & 7.13 \\
\hline Hesperostipa spp. ${ }^{f}$ & G & $\mathrm{P}$ & 32 & 1.25 & 18 & 0.26 & - & - \\
\hline Poa pratensis L. ${ }^{*}$ & G & $\mathrm{P}$ & 31 & 3.36 & 51 & 3.36 & 38 & 3.76 \\
\hline Bromus arvensis $\mathrm{L} . *$ & G & A & 25 & 0.34 & 31 & 0.14 & 13 & 0.01 \\
\hline $\begin{array}{l}\text { Lactuca tatarica (L.) C.A. Mey. Var. } \\
\text { pulchella (Pursh) Breitung }\end{array}$ & $\mathrm{F}$ & A & 22 & 0.08 & 6 & 0.03 & 13 & 0.06 \\
\hline Plantago patagonica Jacq. & $\mathrm{F}$ & A & 22 & 0.06 & 8 & 0.03 & - & - \\
\hline $\begin{array}{l}\text { Bouteloua gracilis (Willd. ex Kunth) } \\
\text { Lag. ex Griffiths }\end{array}$ & G & $\mathrm{P}$ & 20 & 0.61 & 8 & 0.01 & - & - \\
\hline Calamovilfa longifolia (Hook.) Scribn. & G & $\mathrm{P}$ & 20 & 2.65 & 2 & 0.10 & 25 & 0.14 \\
\hline Phlox hoodii Richardson & $\mathrm{F}$ & $\mathrm{P}$ & 20 & 0.12 & 14 & 0.07 & - & - \\
\hline Sphaeralcea coccinea (Nutt._ Rydb. & $\mathrm{F}$ & $\mathrm{P}$ & 20 & 0.11 & 22 & 0.06 & - & - \\
\hline Artemisia dracunculus $\mathrm{L}$. & $\mathrm{F}$ & $\mathrm{P}$ & 19 & 0.31 & 2 & 0.02 & - & - \\
\hline Erysimum asperum (Nutt.) DC. & $\mathrm{F}$ & B & 19 & 0.11 & 2 & $0.00^{\mathrm{g}}$ & - & - \\
\hline Grindelia squarrosa (Pursh) Dunal & $\mathrm{F}$ & A & 19 & 0.50 & 27 & 1.30 & 13 & 0.01 \\
\hline Vicia Americana Muhl. ex Willd. & $\mathrm{F}$ & $\mathrm{P}$ & 19 & 1.19 & 10 & 0.01 & - & - \\
\hline Achillea millefolium $\mathrm{L}$. & $\mathrm{F}$ & $\mathrm{P}$ & 17 & 0.25 & 43 & 0.69 & 50 & 0.16 \\
\hline Artemisia frigida Willd. & $\mathrm{F}$ & $\mathrm{P}$ & 17 & 0.12 & 10 & 0.05 & - & - \\
\hline Taraxacum officinale F.H. Wigg * & $\mathrm{F}$ & $\mathrm{P}$ & 17 & 0.02 & 6 & 0.01 & - & - \\
\hline Opuntia fragilis (Nutt.) Haw & $\mathrm{Su}$ & $\mathrm{P}$ & 15 & 0.02 & 2 & 0.00 & - & - \\
\hline Bouteloua dactyloides (Nutt.) J.T. Columbus & G & $\mathrm{P}$ & 14 & 0.16 & 6 & 0.06 & - & - \\
\hline Camelina microcarpa Andrz. ex. DC.* & $\mathrm{F}$ & A & 14 & 0.01 & 49 & 0.49 & 50 & 0.29 \\
\hline Opuntia sp. & $\mathrm{Su}$ & $\mathrm{P}$ & 14 & 0.11 & 2 & 0.02 & - & - \\
\hline Cirsium undulatum (Nutt.) Spreng. & $\mathrm{F}$ & $\mathrm{P}$ & 12 & 0.09 & 14 & 0.11 & - & - \\
\hline Tetraneuris acaulis (Pursh) Greene var. acaulis & $\mathrm{F}$ & $\mathrm{P}$ & 12 & 0.31 & 4 & 0.02 & - & - \\
\hline Artemisia ludoviciana Nutt. & $\mathrm{F}$ & $\mathrm{P}$ & 10 & 0.46 & 8 & 0.23 & 13 & 0.01 \\
\hline Lygodesmia juncea (Pursh) D. Don ex Hook. & $\mathrm{F}$ & $\mathrm{P}$ & 10 & 0.01 & 6 & 0.01 & - & - \\
\hline
\end{tabular}




\begin{tabular}{|c|c|c|c|c|c|c|c|c|}
\hline Penstemon sp. & $\mathrm{F}$ & $\mathrm{P}$ & 10 & 0.07 & 2 & 0.00 & - & - \\
\hline Potentilla pensylvanica L. & $\mathrm{F}$ & $\mathrm{P}$ & 10 & 1.62 & - & - & - & - \\
\hline Hedeoma hispida Pursh & $\mathrm{F}$ & $\mathrm{P}$ & 8 & 0.01 & 2 & 0.02 & - & - \\
\hline Oxytropis lambertii Pursh & $\mathrm{F}$ & $\mathrm{P}$ & 8 & 0.05 & 6 & 0.02 & - & - \\
\hline Chenopodium rubrum L. & $\mathrm{F}$ & $\mathrm{A}$ & 7 & 0.05 & 2 & 0.00 & - & - \\
\hline Dalea purpurea Vent. & $\mathrm{F}$ & $\mathrm{P}$ & 7 & 0.09 & 4 & 0.00 & - & - \\
\hline $\begin{array}{l}\text { Vulpia octoflora (Walter) } \\
\text { Rydb. var. glauca (Nutt.) Fernald }\end{array}$ & G & A & 7 & 0.17 & 2 & 0.06 & - & - \\
\hline Pediomelum argophyllum (Pursh) J. Grimes & $\mathrm{F}$ & $\mathrm{P}$ & 7 & 0.01 & 6 & 0.02 & - & - \\
\hline Echinacea angustifolia DC. & $\mathrm{F}$ & $\mathrm{P}$ & 5 & 0.04 & 6 & 0.02 & - & - \\
\hline Lappula occidentalis (S. Watson) Greene) & $\mathrm{F}$ & A & 5 & 0.02 & - & - & - & - \\
\hline Pediomelum esculentum (Pursh) Rydb. & $\mathrm{F}$ & $\mathrm{P}$ & 5 & 0.01 & - & - & - & - \\
\hline Carex duriuscula C.A. Mey. & $\mathrm{F}$ & A & 3 & 0.53 & - & - & - & - \\
\hline Escobaria missouriensis (Sweet) D.R. Hunt & $\mathrm{Su}$ & $\mathrm{P}$ & 3 & 0.00 & 4 & 0.02 & 13 & 0.01 \\
\hline Distichlis spicata Greene & G & $\mathrm{P}$ & 3 & 0.03 & 4 & 0.06 & - & - \\
\hline Ellisia nyctelea (L.) L. & $\mathrm{F}$ & A & 3 & 0.00 & 2 & 0.00 & - & - \\
\hline Festuca sp. & G & $\mathrm{P}$ & 3 & 0.01 & 8 & 0.16 & - & - \\
\hline $\begin{array}{l}\text { Oenothera suffrutescens (Ser.) } \\
\text { W. L. Wagner \& Hoch }\end{array}$ & $\mathrm{F}$ & $\mathrm{P}$ & 3 & 0.00 & 2 & 0.00 & - & - \\
\hline Liatris punctata Hook. & $\mathrm{F}$ & $\mathrm{P}$ & 3 & 0.01 & - & - & - & - \\
\hline Rosa woodsii Lindl. & $\mathrm{S}$ & $\mathrm{P}$ & 3 & 0.04 & 6 & 0.04 & - & - \\
\hline Solidago sp. & $\mathrm{F}$ & $\mathrm{P}$ & 3 & 0.04 & 2 & 0.02 & - & - \\
\hline Bromus inermis Leyss. ${ }^{*}$ & G & $\mathrm{P}$ & 2 & 0.12 & 2 & 0.10 & - & - \\
\hline Coryphanthasp. & $\mathrm{Su}$ & $\mathrm{P}$ & 2 & 0.01 & - & - & - & - \\
\hline Descurainia pinnata (Walter) Britton & $\mathrm{F}$ & A & 2 & 0.00 & 18 & 0.06 & 50 & 0.33 \\
\hline $\begin{array}{c}\text { Mirabilis laevis (Benth.) } \\
\text { Curran var. villosa (Kellogg) Spellenb. }\end{array}$ & $\mathrm{F}$ & $\mathrm{P}$ & 2 & 0.00 & - & - & - & - \\
\hline Tragopogon dubius Scop. ${ }^{*}$ & $\mathrm{~F}$ & B & 2 & 0.00 & 8 & 0.01 & - & - \\
\hline Yucca glauca Nutt. & $\mathrm{F}$ & A & 2 & 0.00 & - & - & - & - \\
\hline Eriogonum flavum Nutt. & $\mathrm{F}$ & $\mathrm{P}$ & - & - & 8 & 0.09 & - & - \\
\hline Convolvulus arvensis $\mathrm{L} . *$ & $\mathrm{~F}$ & $\mathrm{P}$ & - & - & 2 & 0.00 & 13 & 0.01 \\
\hline Viola nuttallii Pursh & $\mathrm{F}$ & $\mathrm{P}$ & - & - & 2 & 0.00 & - & - \\
\hline Unknown forb & $\mathrm{F}$ & & 2 & 0.17 & - & - & - & - \\
\hline
\end{tabular}

${ }^{a}$ Class I $=0 \%$ alfalfa cover, Class II $=0.1$ to $50 \%$ alfalfa cover, Class III $>50 \%$ alfalfa cover. ${ }^{b}$ Life Form: Forb (F), Graminoid (G), Shrub (S), and Succulent (Su). ${ }^{\circ}$ Life Span: Annual (A), Biennial (B), and Perennial (P). ${ }^{\mathrm{d}}$ Frequency is the percent of quadrats in the same class in which a species occurs. Bolded values indicate non-alfalfa species frequency greater than $50 \% .{ }^{e}$ Cover is the mean percent cover for all quadrats in the same class in which a species occurs. ${ }^{f}$ Includes Nassella viridula (Trin.) Barkworth, Hesperostipa comata (Trin. \& Rupr.) Barkworth, and Hesperostipa spartea (Trin.) Barkworth. ${ }^{8}$ Cover of $0.00 \%$ is less than $0.01 \%$ but greater than $0 \%$. 


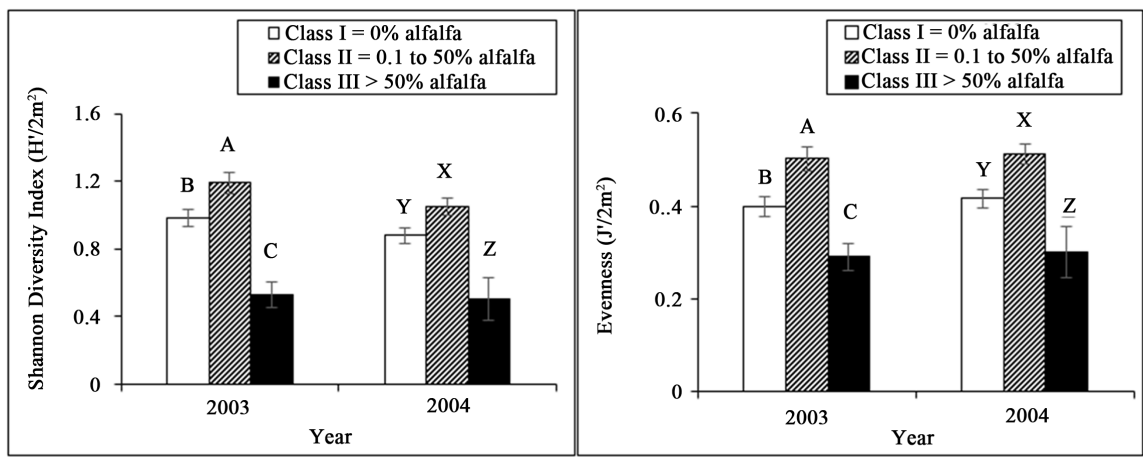

(a)

(b)

Figure 3. Relationship between percent naturalized yellow-flowered alfalfa on species diversity in the Grand River National Grassland near Lodgepole, SD for (a) Shannon Wiener Diversity Index and (b) Evenness. Bars indicate standard errors. Different letters within year represent a significant difference at $P<0.05$.

Plots that had high YFA cover (i.e., Class III) were generally located in swales ( $\mathrm{N}$ site) and toeslope ( $\mathrm{S}$ site) areas. Total biomass increased as YFA cover increased $(P<0.05)$ (Figure $4(\mathrm{a})$ ). Total biomass of YFA in cover Class III was 2.6 times greater than Class I. In contrast, the non-YFA biomass contribution to total biomass declined from $100 \%$ to $85 \%$ to $30 \%$ with increasing YFA cover class $(P<0.05)$ (Figure 4(b)).

Significant difference for soil texture occurred among the three vegetation classes. Soil that supported class I (alfalfa absent) had higher sand content (54\% and 56\%) than class II and III (34\% - 39\%). Conversely, soils that supported class II and III alfalfa cover had higher clay content $(25 \%-31 \%)$ than class I $(19 \%-21 \%)$. Soil texture within each cover class was essentially the same between the $0-15 \mathrm{~cm}$ and $15-30 \mathrm{~cm}$ depths.

A strong positive linear association existed between YFA cover and alfalfa soil seedbank density ( $\mathrm{r}$ values ranged from 0.76 to $0.82, P<0.0001$ ) (Figure 5 ). Seed density of YFA ranged from 0 to over 40,000 seeds $\mathrm{m}^{-2}$ for individual plots with a mean of 4400 seeds $\mathrm{m}^{-2}$ (equivalent to $4.4 \times 10^{7}$ seeds ha ${ }^{-1}$ ). Greater than $99 \%$ of YFA seeds collected from the soil seed bank during 2004, 2005, and 2006 were viable (Table 2). However, less than $4 \%$ germinated under standard laboratory conditions, indicating an extremely high level of hard seed in this YFA population.

\section{Discussion}

YFA can be established in crested wheatgrass and native grassland using interseeding [2] [4] or transplant methods [12]. However, the current study demonstrated, as pointed out by Smith [2], that YFA can also establish, survive, and spread naturally in native mixed-grass prairie, in this study under USDA-Forest Service grazing schedules. Naturalized YFA, by being restricted to swale and toeslope regions, was strongly associated with increased total biomass production; however, high cover of YFA was also associated with reduced species 


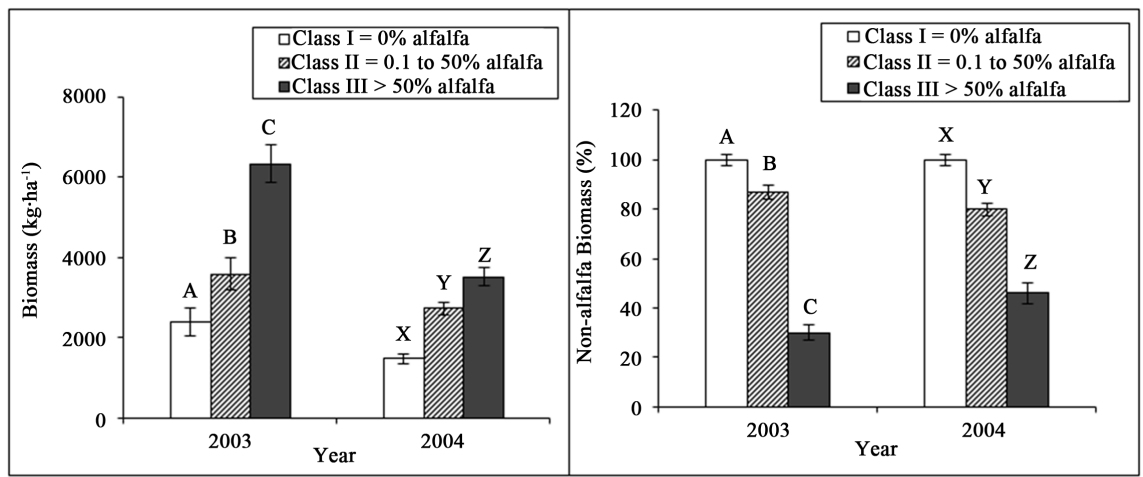

(a)

(b)

Figure 4. Relationship of percent cover of naturalized yellow-flowered alfalfa to (a) total biomass and (b) percentage of total biomass from non-alfalfa species on the Grand River National Grassland near Lodgepole, SD. Bars indicate standard errors. Different letters within year represent a significant difference at $P<0.05$.
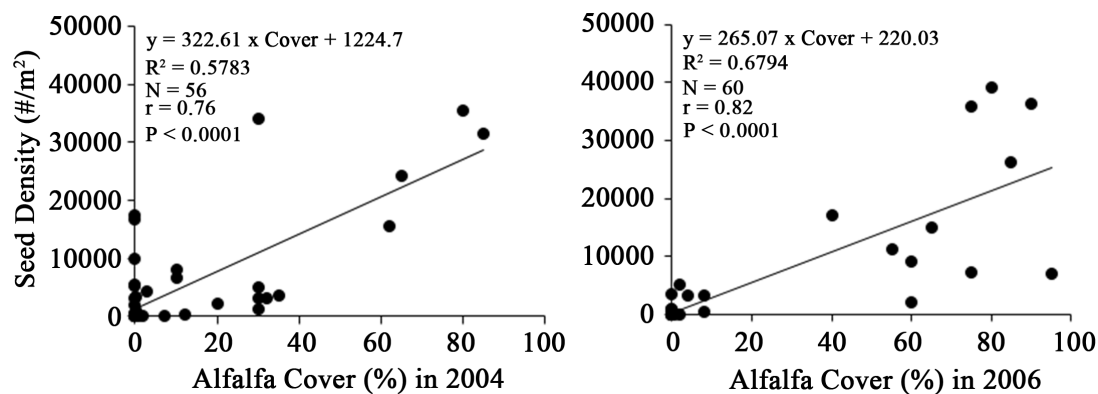

Figure 5. Relationship between yellow-flowered alfalfa soil seed bank density and alfalfa cover on the Grand River National Grassland near Lodgepole, SD in 2004 and 2006.

Table 2. Germination and viability of seeds collected from the alfalfa soil seed bank from Pasture 4B on the Grand River National Grassland near Lodgepole, SD (4 replications/site/year, 100 seeds/replication).

\begin{tabular}{ccccc}
\hline \multirow{2}{*}{ Year } & \multicolumn{2}{c}{ Germination (\%) } & \multicolumn{2}{c}{ Viability (\%) } \\
\cline { 2 - 5 } & North Site & South Site & North Site & South Site \\
\hline 2004 & 1.0 & 8.2 & 100 & 99.7 \\
2005 & 3.5 & 7.7 & 99.7 & 99.5 \\
2006 & 4.0 & 2.7 & 99.5 & 99.5 \\
\hline
\end{tabular}

richness and lower biomass production from native species. Generally, these areas have more favorable water and nutrient conditions relative to the sandy loam soils of the drier backslopes and shoulders, and thus are more suitable for the establishment and growth of alfalfa [33].

Although the difference in total biomass production within classes between years ranged from $24 \%$ to $45 \%$, the relative contribution of YFA and non-YFA components to biomass between years, irrespective of the class type of the vegetation, were consistent (Figure 4(a) and Figure 4(b)). The difference in precipitation (Figure 6) between $2003(457 \mathrm{~mm})$ and $2004(325 \mathrm{~mm})$ provided an opportunity to evaluate the impact of YFA on total biomass production (Figure 7). 


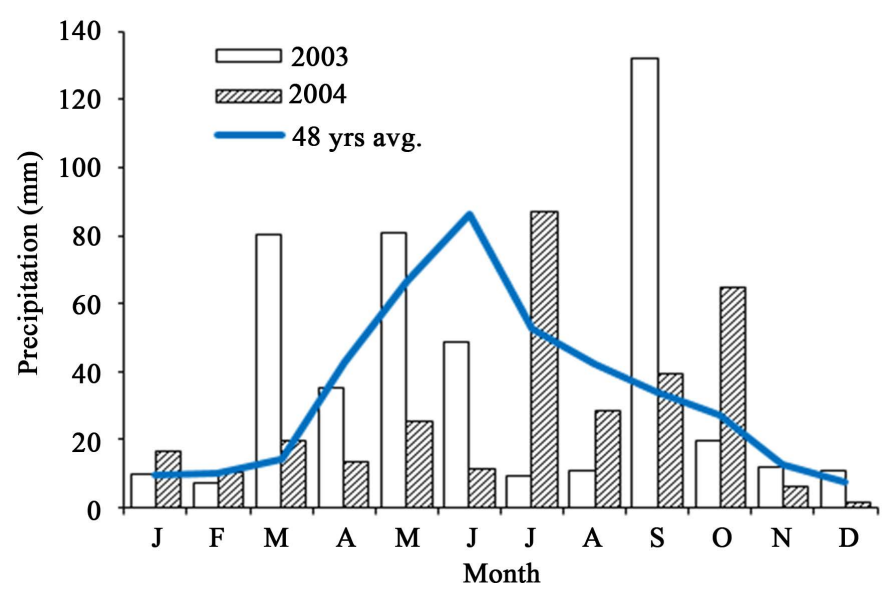

Figure 6. Monthly precipitation totals in 2003 and 2004 compared with 48-year average at Hettinger, ND (nearest weather station to study site).

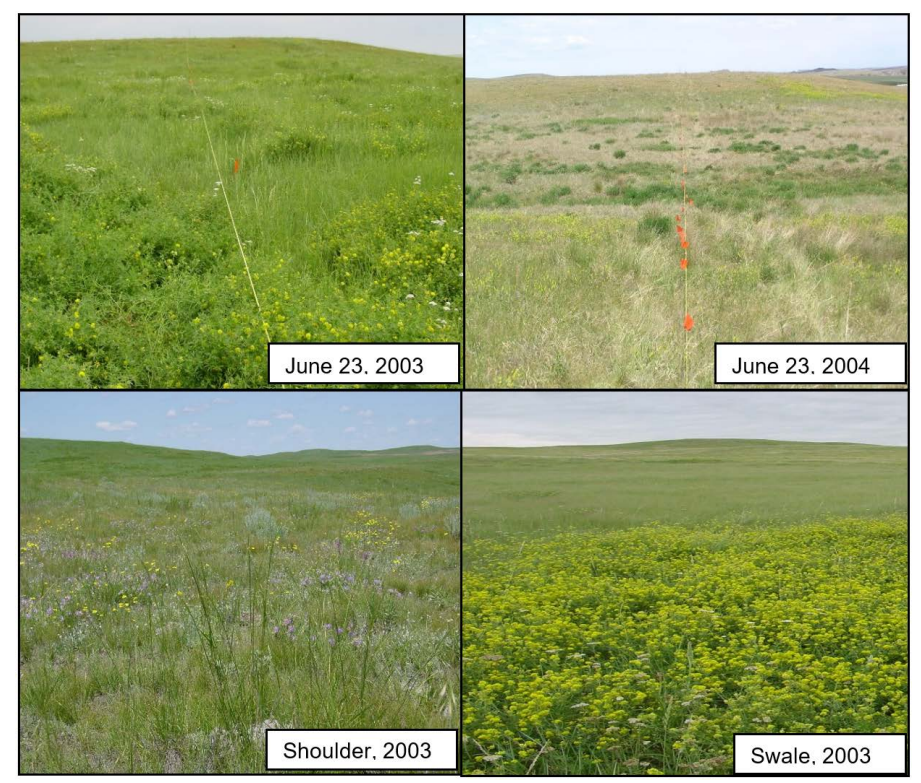

Figure 7. Distribution of naturalized YFA showing temporal (upper, 2003 vs. 2004) and spatial (lower, shoulder vs. swale in 2003) variations on the Grand River National Grassland.

It was evident that the benefit of the YFA component to increased biomass was stable across both years.

Hansen [34] pointed out that alfalfa on the Grand River National Grassland was most abundant in areas that had most likely been privately farmed up to the mid-1930s, but were then reseeded to predominately crested wheatgrass by the Soil Conservation Service under the Bankhead-Jones Farm Tenet Act. Thereafter, during the 1950s the federally reclaimed land was turned over to the USDA-Forest Service for permanent management. Therefore, it is our assertion that present YFA dominated plant communities on GRNG are a ramification of previous land utilization that replaced the native vegetation with annual cropping systems for several decades prior to reseeding introduced species in re- 
sponse to economic hardships and ecological catastrophes, such as drought and erosion.

Over the past 15 years, similar to what was reported by Samuel, et al. [35] for secondary succession areas dominated by western wheatgrass in rangelands of Wyoming, we have observed closer selective grazing in the YFA and crested wheatgrass dominated areas compared with the higher diversity native vegetation on adjacent backslopes and shoulders. Under the current grazing management practices of the USDA-Forest Service, the YFA dominated areas appear to be compositionally stable, more productive, and no threat to the surrounding natural biodiversity.

Seeds collected from soil cores in June 2004, November 2005, and June 2006 were likely not produced on-site during those years. In 2004, larvae of the alfalfa weevil (Hypera postica Gyllenhall) destroyed nearly $100 \%$ of the axillary branches containing floral meristems from the initial growth cycle in the spring. In 2005, the pasture was grazed heavily during flowering, which also precluded on-site seed production. In 2006, sampling was before mature seeds were produced. However, this does not rule out grazing livestock having brought ingested seeds to the study site from adjacent privately owned pastures during that three-year period.

Seed bank of YFA was not uniformly distributed across the landscape. Seeds were concentrated in areas of high YFA cover in swales ( $\mathrm{N}$ site) and toeslope ( $\mathrm{S}$ site) areas and were infrequent in plant communities lacking YFA, such as the threadleaf sedge community on shoulders. We observed seeds and young seedlings in cattle feces on shoulders and backslopes; however, we rarely observed seedling establishment in those areas. Thus, recruitment of YFA in areas of low YFA cover is possible but unlikely. The swales and toeslope areas dominated by YFA contained a large reservoir of viable YFA seeds. We observed alfalfa seedlings that ranged from cotyledon stage to 5-trifoliate leaf stage under the canopy in about $40 \%$ of the total quadrats comprising Class II and III areas during vegetation sampling in 2003; whereas, no seedlings were found in Class I quadrats. The highest seedling density was 120 seedlings $\mathrm{m}^{-2}$. Most were etiolated due to shading by the established plants. In contrast, no seedlings were found in any of the quadrats in 2004, presumably because of drought, but also insect feeding. This population has, through periodic seed production and an inherent high level of hard seed, the capacity for population maintenance and potential expansion on favorable landscape positions.

Seed of YFA introduced to the northern Great Plains by N.E. Hansen was first planted near the present day GRNG by Charles Smith in 1915. In the early 1970s, Norman Smith, grandnephew of Charles Smith, noticed several small patches of YFA that had spread from the original 1915 planting on his ranch. Smith began planting seed from those patches in the early 1980s in tilled cropland and native rangeland. By 2000 [3], Smith had about 200 hectares of alfalfa that traced back to Hansen's 1915 introduction through mostly natural recruitment from plants established by interseeding [2]. 
The seed source of the GRNG population in Pasture 4B is undoubtedly the Smith Ranch population [36]. The ranch borders Pasture $4 \mathrm{~B}$ for about $5 \mathrm{~km}$ on the west. The drainage pattern in that area runs from west to east. The Smith Ranch also has long-standing annual grazing rights from the USDA Forest Service for Pasture 4B. Thus, dissemination of seed from the Smith Ranch to Pasture $4 \mathrm{~B}$ is most likely due to both abiotic and biotic factors [2]. Because of its proximity to the Smith Ranch, Pasture 4B is uniquely located to have received seed from a YFA population that demonstrated reseeding ability and, once established, could maintain a breeding population.

Our sampling sites on the GRNG were within $2 \mathrm{~km}$ of the Smith Ranch pastures containing YFA. Smith was successful in establishing yellow-flowered alfalfa by interseeding on mid- and upper backslope positions on his ranch. This was facilitated by his invention of an interseeder that created a furrow that disturbed and stymied the native vegetation and created a depression to hold moisture around the seed and developing seedlings. In contrast, natural recruitment on the GRNG on mid- and upper backslopes relies on seeds being placed in a natural microenvironment, disturbed or undisturbed, that will provide conditions suitable for germination, seedling establishment, and subsequent perennation.

A section of land containing a valley terrace within $2 \mathrm{~km}$ of our study area in Pasture $4 \mathrm{~B}$ was farmed during the early $20^{\text {th }}$ century up to the 1950s [36], with the notable exception of those areas that were too steep or too rocky. During the 1950s the farmland was planted to mainly crested wheatgrass. The lines of separation between farmland planted to crested wheatgrass and native range were still evident at the initiation of this study. Kopp [36] conducted a floristic and vegetational analysis of the GRNG and rated the crested wheatgrass reseeded areas of marginal natural quality based on Coefficients of Conservatism, Floristic Quality Index, and proportion (19.4\%) of exotic species. He also pointed out the similarity between YFA on Pasture 4B and crested wheatgrass regarding their potential invasiveness; however, he determined there was no evidence that crested wheatgrass was spreading out from the original plantings of the mid $20^{\text {th }}$ century.

Hansen and Hoffman [37] conducted a vegetational analysis of the GRNG during 1982-1984, focusing on the least disturbed areas. They reported the most common steppe plant association in three sections of land within $10 \mathrm{~km}$ east-northeast of our study area was the Hesperostipa comata-Carex filifolia habitat type. They found very low frequency of yellow sweetclover [Melilotus officinalis (L.) Lam.] and no occurrence of YFA.

This study showed that from unintentional introduction(s), subsequent naturalization of a highly adapted YFA in grazed mixed-grass prairie in the semiarid northern Great Plains was largely limited to lower landscape positions, which were dominated by introduced species (i.e., crested wheatgrass), and were the most favorable areas for its natural propagation (Figure 7). Mortenson, et al. [3] found interseeded plantings of alfalfa made on the Smith Ranch in 1965 or 1987 
had established uniform stands between rows by 2001 . However, plantings made in 1998 showed no between-row spreading. Based on floristic observations on record in the GRNG District Ranger Headquarters in Lemmon, SD, we surmise the naturalization of YFA in Pasture $4 \mathrm{~B}$ has occurred over the past 35 or so years. We conducted a reconnaissance of Pasture 4B in July 2018 and saw no visually perceptible difference from 2004 , in terms of alfalfa stand density at the various topographic positions across the heterogeneous landscape.

\section{Acknowledgements}

Dr. Kevin Kephart, former Director of the SDAES, was particularly supportive of the research. We also appreciate the support of US Forest Service personnel: Darla Lenz (Bismarck) and Jack Isaacs, Curt Hansen, and Mary Haase (Lemmon). We acknowledge Matt Stoltenberg, Kyle Brehe, Gang Li, Xiangfan Yin, and Céline Sicard for their assistance in the field and laboratory. Thanks are also extended to Ronnie Parmely and Dennis Ruhlman, SDSU Seed Testing Laboratory, and Beverly Klein, SDSU Soil Chemistry Laboratory.

\section{Funding}

Research was funded in part by the South Dakota Agricultural Experiment Station through USDA-NIFA and US Forest Service Dakota Prairie Grasslands, Bismarck, ND and Lemmon, SD.

\section{Conflicts of Interest}

The authors declare no conflicts of interest regarding the publication of this paper.

\section{References}

[1] Lorenz, R.J., Ries, R.E., Cooper, C.S., Townsend, C.E. and Rumbaugh, M.D. (1982) Preface. In: Alfalfa for Dryland Grazing, US Department of Agriculture, Washington DC, Agricultural Research Service, Agriculture Information Bulletin No. 444.

[2] Smith, N.G. (1997) Yellow-Blossomed Alfalfa on Rangeland in South Dakota. Rangelands, 19, 24-25.

[3] Mortenson, M.C., Schuman, G.E., Ingram, L.J., Nayigibugu, V. and Hess, B.W. (2005) Forage Production and Quality of a Mixed-Grass Rangeland Interseeded with Medicago sativa ssp. falcata. Rangeland Ecology and Management, 58, 505-513. https://doi.org/10.2111/1551-5028(2005)58[505:FPAQOA]2.0.CO;2

[4] Misar, C.G., Xu, L., Gates, R.N., Boe, A., Johnson, P.S., Schauer, C.S., Rickertsen, J.R. and Stroup, W.W. (2016) Establishment and Persistence of Yellow-Flowered Alfalfa No-Till Interseeded into Crested Wheatgrass Stands. Agronomy Journal, 108, 141-150. https://doi.org/10.2134/agronj2015.0271

[5] Bolton, J.L., Goplen, B.P. and Baenziger, H. (1972) World Distribution and Historical Developments. In: Hanson, C.H. Ed., Alfalfa Science and Technology, Agronomy Monograph No. 15, ASA, Madison, 1-34.

[6] Rumbaugh, M.D. (1982) Reseeding by Eight Alfalfa Populations in a Semiarid Pasture. Journal of Range Management, 35, 84-86. https://doi.org/10.2307/3898526 
[7] Hansen, N.E. (1909) The Wild Alfalfas and Clovers of Siberia, with a Perspective View of Alfalfas of the World. USDA Bur. Plant Ind. Bull. 150. https://doi.org/10.5962/bhl.title.43419

[8] Oakley, R.A. and Garver, S. (1917) Medicago falcata, a Yellow-Flowered Alfalfa. USDA Bur. Plant Indust. Bull. 428. https://doi.org/10.5962/bhl.title.108577

[9] Heinrichs, D.H. (1975) Potential of Legumes for Rangelands. In: Campbell, R.S. and Herbel, C.H., Eds., Improved Range Plants, Range Symposium Series 1, Society for Range Management, Denver, 50-61.

[10] Berdahl, J.D., Wilton, A.C. and Frank, A.B. (1989) Survival and Agronomic Performance of 25 Alfalfa Cultivars and Strains Interseeded into Rangeland. Journal of Range Management, 42, 312-316. https://doi.org/10.2307/3899501

[11] Hanson, A., Xu, L., Boe, A., Johnson, P.S., Gates, R.N. and Wu, Y. (2015) Identification and Characterization of Drought Tolerant Alfalfa (Medicago sativa subsp. falcata) Germplasm. Proceedings of the South Dakota Academy of Science, 94, 263-272.

[12] Misar, C.G., Xu, L., Gates, R.N., Boe, A. and Johnson, P.S. (2015) Stand Persistence and Forage Yield of 11 Alfalfa (Medicago sativa) Populations in Semiarid Rangeland. Rangeland Ecology \& Management, 68, 79-85.

https://doi.org/10.1016/j.rama.2014.12.012

[13] Berdahl, J.D., Wilton, A.C., Lorenz, R.J. and Frank, A.B. (1986) Alfalfa Survival and Vigor in Rangeland Grazed by Sheep. Journal of Range Management, 39, 59-62. https://doi.org/10.2307/3899688

[14] Mortenson, M.C., Schuman, G.E. and Ingram, L.J. (2004) Carbon Sequestration in Rangelands Interseeded with Yellow-Flowering Alfalfa (Medicago sativa ssp. falcata). Environmental Management, 33, S475-S481. https://doi.org/10.1007/s00267-003-9155-9

[15] Schuman, G.E., Ingram, L.J. and Parkin, T.B. (2004) Nitrous Oxide Emissions from a Northern Mixed-Grass Rangeland Interseeded with Yellow-Flowering Alfalfa (Medicago sativa ssp. falcata). 57 th Annual Meeting on Rangelands in Transition, Salt Lake City, 24-30 January 2004, 180.

[16] Richardson, D.M., Pysek, P., Rejmanek, M., Barbour, M.G., Panetta, F.D. and West, C.J. (2000) Naturalization and Invasion of Alien Plants: Concepts and Definitions. Diversity and Distribution, 6, 93-107. https://doi.org/10.1046/j.1472-4642.2000.00083.x

[17] Pimentel, D., Lach, L., Zuniga, R. and Morrison, D. (2000) Environmental and Economic Costs of Non-Indigenous Species in the United States. BioScience, 50, 53-65. https://doi.org/10.1641/0006-3568(2000)050[0053:EAECON]2.3.CO;2

[18] Pellant, M. and Lysne, C.R. (2005) Strategies to Enhance Plant Structure and Diversity in Crested Wheatgrass Seedings. USDA Forest Service Proceedings RMRS-P-38.

[19] Boe, A., Bortnem, R., Higgins, K.F., Kruse, A.D., Kephart, K.D. and Selman, S. (1998) Breeding Yellow-Flowsered Alfalfa for Combined Wildlife Habitat and Forage Purposes. South Dakota State University, Brookings, South Dakota Agricultural Experiment Station B 727, 12 p.

[20] Lowe, T.W. (1975) Resource Inventory of the Grand River Basin. South Dakota Department of Natural Resources, Pierre, $194 \mathrm{p}$.

[21] High Plains Regional Climate Center (2012) Historical Climate Data Summaries. https://hprcc.unl.edu/climatesummaries.php

[22] North Dakota Agricultural Weather Network (2012) Monthly Report. https://ndawn.ndsu.nodak.edu//monthly-report-form.html 
[23] USDA-NRCS-NSSC (2012) Field Book for Describing and Sampling Soils. Version 3.0.

[24] Sims, P.L. and Risser, P.G. (2000) Grasslands. In: Barbour, M.G. and Billings, W.D., Eds., North American Terrestrial Vegetation, Second Edition, Cambridge University Press, Cambridge, 323-356.

[25] US Forest Service Dakota Prairie Grasslands (2014). http://www.fs.usda.gov/lan.dpg.landmanagement

[26] USDA PLANTS Database (2019). http://www.plants.usda.gov

[27] USDA-NRCS-NSSC (2004) Soil Survey Laboratory Methods Manual. Soil Survey Investigations Report No. 42 Version 4.0.

[28] AOSA (2014) Association of Official Seed Analysts Rules.

[29] Daubenmire, R. (1968) A Canopy-Coverage Method of Vegetational Analysis. Northwest Science, 33, 43-64.

[30] Barbour, M.G., Burk, J.H., Pitts, W.D., Gilliam, F.S. and Schwrtz, M.W. (1999) Terrestrial Plant Ecology. 3rd Edition, The Benjamin/Cummings, an Imprint of Addison Wesley Longman, Inc., Boston.

[31] Magurran, A.E. (2004) Measuring Biological Diversity. Blackwell Publishing, Hoboken.

[32] SAS Institute (1990) SAS/STAT User's Guide, Version 6. SAS Institute, Inc., Cary.

[33] Barnes, D.K. and Sheaffer, C.C. (1985) Alfalfa. In: Heath, M.E., Barnes, R.F. and Metcalfe, D.S., Eds., Forages: The Science of Grassland Agriculture, 4th Edition, Iowa State Univ. Press, Ames, 89-97.

[34] Hansen, K. (2008) Plants of the Grand River and Cedar River National Grasslands: 2008. USDA-Forest Service. Dakota Prairie Grasslands. 2600-Botany. 56 p.

[35] Samuel, M.J. and Hart, R.H. (1994) Sixty-One Years of Secondary Succession on Rangelands of the Wyoming High Plains. Journal of Range Management, 47, 184-191. https://doi.org/10.2307/4003013

[36] Kopp, C.W. (2004) Floristic and Vegetational Analysis of the Grand River National Grassland. M.S. Thesis, South Dakota State University, Brookings.

[37] Hansen, P.L. and Hoffman, G.R. (1988) The Vegetation of the Grand River/Cedar River, Sioux, and Ashland Districts of the Custer National Forest: A Habitat Type Classification. USDA Forest Service, Rocky Mountain Forest and Range Experiment Station General Technical Report RM-157, Fort Collins.

https://doi.org/10.2737/RM-GTR-157 